\title{
MECHANOCHEMICAL COUPLING IN
}

\author{
FLAGELLA
}

\section{EFFECTS OF VISCOSITY ON MOVEMENT AND ATP-DEPHOSPHORYLATION OF TRITON- DEMEMBRANATED SEA-URCHIN SPERMATOZOA}

\author{
C. J. BROKAW AND T. F. SIMONICK \\ Division of Biology, California Institute of Technology, Pasadena, California 91125, \\ U.S.A.
}

\section{SUM M A R Y}

Improved techniques have been used to measure movement-coupled ATP-dephosphorylation by suspensions of motile, reactivated sea-urchin spermatozoa. The results confirm that the movement-coupled ATP-dephosphorylation is proportional to beat frequency under conditions where the bend angle remains constant. Some observations suggest that the rate of ATPdephosphorylation is also related to the amplitude of sliding between flagellar tubules, but our attempts to measure this relationship have been unsuccessful.

\section{INTRODUCTION}

Previous studies of the effect of viscosity on the rate of ATP-dephosphorylation by demembranated sea-urchin spermatozoa suggested that the movement-dependent ATP-turnover was proportional to the frequency of flagellar beating (Brokaw \& Benedict, I968a; Gibbons \& Gibbons, I972). However, in those studies it was not possible to exclude the possibility that changes in other movement parameters besides the beat frequency might be responsible for the changes in the rate of ATP-dephosphorylation. Improved techniques and methods for reactivating the movement of demembranated sea-urchin spermatozoa (Gibbons \& Gibbons, I972; Brokaw, Josslin $\&$ Bobrow, r974) have now enabled us to carry out more definitive experiments, which solidly confirm the relationship between beat frequency and ATP turnover.

\section{MATERIALS AND METHODS}

\section{Preparation of demembranated spermatozoa}

Spermatozoa of the sea urchin, Strongylocentrotus purpuratus, were collected from the aboral surface of an urchin which had been injected with $0.6 \mathrm{M} \mathrm{KCl}$ to induce shedding of gametes. These spermatozoa were diluted with about 2 vol. of cold $0.5 \mathrm{M} \mathrm{NaCl}$, to give a suspension which was thin enough to sample reproducibly. Sperm concentrations were standardized by diluting a ro- $\mu 1$ sample of the stock sperm suspension with $5.0 \mathrm{ml}$ of $0.5 \mathrm{M} \mathrm{NaCl}$ and measuring the optical density at $540 \mathrm{~nm}$. The sperm concentration in the stock suspension was adjusted until an optical density reading of $0.24-0.26$ was obtained. This stock suspension was stored in an ice bucket and used through a 4-6-h period. Measurements on sperm suspensions following overnight storage in a refrigerator showed no apparent decrease in the quality of the results. 
Sperm counts were carried out on some of the stock sperm suspensions, using a procedure similar to that of Gibbons \& Fronk (1972). A 50- $\mu$ l capillary pipette was used to transfer a $50-\mu \mathrm{l}$ sample of the stock suspension to a dry 4-ml test tube. This sample was fixed by adding $0.45 \mathrm{ml}$ of $5 \%$ formaldehyde in $0.5 \mathrm{M} \mathrm{NaCl}$. After 20-30 min, two successive $\mathrm{r}: 200$ dilutions with distilled water were carried out. A small drop $(5-15 \mu \mathrm{l})$ of the final dilution was placed on a weighed microscope slide, and the slide was immediately reweighed to determine the volume of the drop. The drop was evaporated to dryness, and all the spermatozoa in the drop (usually about 500 ) were counted, using a phase contrast microscope with a $\times 25$ objective. Two or three counts were carried out on each diluted sample.

Demembranated spermatozoa were prepared when needed by transferring a 50- $\mu 1$ sample of the stock sperm suspension to a $5-\mathrm{ml}$ test tube with a $50-\mu \mathrm{l}$ capillary pipette, and adding $\mathrm{I} \cdot \circ \mathrm{ml}$ of extraction solution, at $16^{\circ} \mathrm{C}$. The extraction solution contained $0.15 \mathrm{M} \mathrm{KCl,2} \cdot 0 \mathrm{~mm} \mathrm{MgSO}$, $2.0 \mathrm{mM} \mathrm{CaCl}, 2.0 \mathrm{mM}$ Tris buffer, $2.0 \mathrm{~mm}$ dithiothreitol (Sigma Chemical Co.), $0.5 \mathrm{~mm}$ EDTA, and $0.04 \%$ (vol/vol) Triton X-I00 (Sigma). Its $\mathrm{pH}$ was adjusted to 8.2 at $16{ }^{\circ} \mathrm{C}$. Spermatozoa were extracted for $30 \mathrm{~s}$ before being added to reactivation solution.

\section{$p H$-stat measurements of ATP-dephosphorylation}

Rates of ATP-dephosphorylation in suspensions of reactivated spermatozoa were obtained from measurements of $\mathrm{H}^{+}$ion release during the reaction, using recording $\mathrm{pH}$-stats $(\mathrm{E}$. $\mathrm{H}$. Sargent \& Co.). The pH-stat methods were similar to those used in previous work (Brokaw $\&$ Benedict, $1968 a, b$ ), except for the following modifications: Measurements were made at $\mathrm{pH} 8.4$, which gave optimum motility, and nearly complete release of inorganic phosphate in the $\mathrm{HPO}_{4}{ }^{2}$ ion form, so that the rate of ATP-dephosphorylation was essentially identical to the rate of $\mathrm{H}^{+}$ion release. $\mathrm{CO}_{2}$ absorption was eliminated by maintaining an atmosphere of $\mathrm{N}_{2}$ gas over the surface of the assay solution. The Beckman no. 41239 reference electrode was isolated from the assay solution by a salt bridge made from a $4-\mathrm{cm}$ medial portion of a disposable Pasteur pipette fastened over the end of the electrode with a rubber sleeve. The narrow end of the bridge was filled with a short $(0.5-1.0 \mathrm{~cm})$ plug of $4 \%$ agar in $3 \mathrm{M} \mathrm{KCl}$, which formed the junction with the assay solution. The remainder of the bridge was filled with $3 \mathrm{M} \mathrm{KCl}$, which formed the junction with the tip of the reference electrode. This arrangement appeared to give improved motility, possibly because contamination by $\mathrm{Hg}^{+}$ions was prevented, and also gave improved reference electrode stability, probably because the reference electrode junction was isolated from the thioglycollate in the assay solution. Each salt bridge was routinely replaced after 2 rate measurements ( $\mathrm{I}-2 \mathrm{~h}$ in the assay solutions).

Two $\mathrm{pH}$-stats were used in parallel, at $16^{\circ} \mathrm{C}$ and $\mathrm{pH} 8.4$, with $0.005 \mathrm{M} \mathrm{NaOH}$ as titrant. The titrant was standardized at the end of each day, by recording the response to addition of $\mathrm{HCl}$ at $100 \mathrm{~nm} \mathrm{~min}{ }^{-1}$ with a Sage syringe pump. The reactivation solution used for $\mathrm{pH}$-stat assays contained $\circ .15 \mathrm{M} \mathrm{KCl}, 1 . \circ \mathrm{mM} \mathrm{Na}$ thioglycollate (prepared from concentrated thioglycollic acid), $0.2 \mathrm{mM}$ EGTA, $20 \mathrm{mg} \mathrm{ml}^{-1}$ polyethylene glycol (carbowax; Fisher P-162), $8 \mu \mathrm{g} \mathrm{ml}^{-1}$ oligomycin (Calbiochem), and appropriate concentrations of $\mathrm{MgSO}_{4}$ and ATP (BoehringerMannheim). Some of the solutions also contained high molecular weight methyl cellulose (Fisher M-28t), diluted from a $2 \%$ stock solution. For a typical experiment examining the effect of increased viscosity, a $10-\mathrm{ml}$ portion of assay solution without methyl cellulose was put in a reaction beaker on one $\mathrm{pH}-\mathrm{stat}$, and a $10-\mathrm{ml}$ portion of assay solution with methyl cellulose was put on the other $\mathrm{pH}$-stat. After adjusting temperature, $\mathrm{pH}$, and $\mathrm{N}_{2}$ flow, base lines were established for at least $10 \mathrm{~min}$. If necessary, $\mathrm{HCl}$ was added at a rate of 2 or $5 \mathrm{nM} \mathrm{min}-1$ with a Sage syringe pump, in order to obtain a positive base line. There is an immediate burst of $\mathrm{H}^{+}$ production resulting from reaction between $\mathrm{Ca}^{2+}$ and EGTA when extraction solution is added to the reactivation solution. To minimize the $\mathrm{pH}$-stat response to this $\mathrm{H}^{+}$burst an appropriate amount of $\mathrm{NaOH}$ was added to each reaction beaker just before preparing the demembranated sperm sample, and $0.25-\mathrm{ml}$ portions of the demembranated sperm suspension were then added in quick succession to each $\mathrm{pH}$-stat reaction beaker. There was usually a delay of several minutes before initial $\mathrm{pH}$ adjustment was completed and a steady rate of $\mathrm{H}^{+}$production was obtained. Rates for both solutions were measured over a $10-$ min period, as soon as steady rates were established in both solutions. The experiment was then repeated with the order of solu- 
tions on the $\mathrm{pH}$-stats reversed, and the averaged results from the 2 experiments were used to obtain a data point for Fig. I in the Results section.

\section{Motility observations}

After recording of the ATP dephosphorylation rate on the $\mathrm{pH}$-stats had begun, a sample was removed from each reaction beaker, placed on a microscope slide at $16{ }^{\circ} \mathrm{C}$ without a coverglass, and examined microscopically to check the percentage motility and the characteristics of the movement. Data from experiments in which the percentage motility in the absence of methyl cellulose was judged to be less than $90 \%$ were discarded. Beat frequencies of several representative spermatozoa swimming at the upper surface of the drop were measured using stroboscopic illumination, and a visual estimate (Brokaw, I $975 a$ ) of the bend angles of the flagellar waveforms was made. In a few experiments, samples were also removed from the $\mathrm{pH}$-stat reaction beakers for photography to measure bend angles and wavelengths. These samples were diluted with reactivation solution buffered to $\mathrm{pH} 8.4$ with $20 \mathrm{mM}$ Tris buffer. Because of the necessity for dilution and because most of the spermatozoa stick to the coverglass or microscope slide under these conditions, this procedure probably does not provide a significantly better characterization of the bend angles than is obtained by visual observations.

Some independent experiments were carried out under conditions more optimal for measurement and photography of movement parameters. These used solutions of identical composition, except for the addition of $20 \mathrm{mM}$ Tris to buffer the reactivation solutions at $\mathrm{pH} 8 \cdot 4$, but the procedure described previously for gentle treatment of small samples (Brokaw, 1975a) was used. For these measurements, we usually obtained a mean beat frequency at each methyl cellulose concentration by recording the beat frequencies of about 15 spermatozoa in each of two Tritonated sperm preparations. Photographs were made on Tri-X film with an $\mathrm{X}_{40}$ oil immersion objective, giving a final magnification on film of $\mathrm{I} 60 \times$. Illumination for stroboscopic measurements and multiple-flash photographs was obtained from Xenon flash equipment obtained from the Chadwick-Helmuth Co., Monrovia, California.

\section{ATP-dephosphorylation by non-motile spermatozoa}

In order to identify the component of flagellar ATP-dephosphorylation which is coupled to movement, and to identify any effects of methyl cellulose on measured rates of ATP dephosphorylation which might be unrelated to movement, similar $\mathrm{pH}$-stat measurements were carried out with non-motile spermatozoa. Some of these measurements were obtained from experiments with spermatozoa which were broken by vigorous shaking for $40 \mathrm{~s}$ with a Vortex mixer, before adding the extraction solution to the spermatozoa. With this procedure, a typical I-day experiment with a particular sperm sample involved a set of measurements on whole spermatozoa, a set of measurements on broken spermatozoa, and a final set of measurements on whole spermatozoa.

An alternative procedure used in later experiments involved the addition of $2 \mu \mathrm{g}$ of trypsin (Sigma, T8003) to each reaction vessel after recording the rate of $\mathrm{H}^{+}$production by motile spermatozoa. After 3-4 min, the spermatozoa disintegrate and there is a relatively abrupt change in the rate of $\mathrm{H}^{+}$production to a lower level characteristic of the disintegrated, nonmotile spermatozoa (Brokaw \& Simonick, manuscript in preparation). In some of these experiments, $2 \mu \mathrm{g}$ of soybean trypsin inhibitor was added 5 min after adding the trypsin. This trypsin concentration appears to be low enough so that the rate of $\mathrm{H}^{+}$production by tryptic digestion of protein is undetectable, and low enough so that neither activation (Linck, 1973; Ogawa, I973) nor inactivation of the residual flagellar ATPase was detectable during the time period of our measurements. Using this procedure, we could usually obtain 3 sets of measurements on both motile and non-motile spermatozoa from a I-day experiment with a particular sperm sample.

\section{Viscosity measurements}

Capillary viscosimeters were used to measure the viscosities of the reactivation solutions (Brokaw, I 966 ). For the $\mathrm{pH}$-stat experiments, the viscosity measurements were carried out with portions of the assay solution containing spermatozoa, after completion of a $\mathrm{pH}$-stat measure- 
ment. For the independent observations on motility, the viscosity measurements were carried out on reactivation solutions without spermatozoa. The differences between these measurements were found to be negligibli.

\section{RESULTS}

\section{Measurements of ATP dephosphorylation}

The first series of experiments was carried out with reactivation solutions containing $1.3 \mathrm{~mm} \mathrm{MgSO}_{4}$ and $0.33 \mathrm{~mm}$ ATP, using methyl cellulose concentrations of 0.1 , $0.2,0.4,0.6,0.8$ and $\mathrm{x} \cdot 0 \%$. Data obtained from 22 sperm samples, with unbroken and broken sperm preparations, are shown in Fig. I A. The data points shown in Fig. I

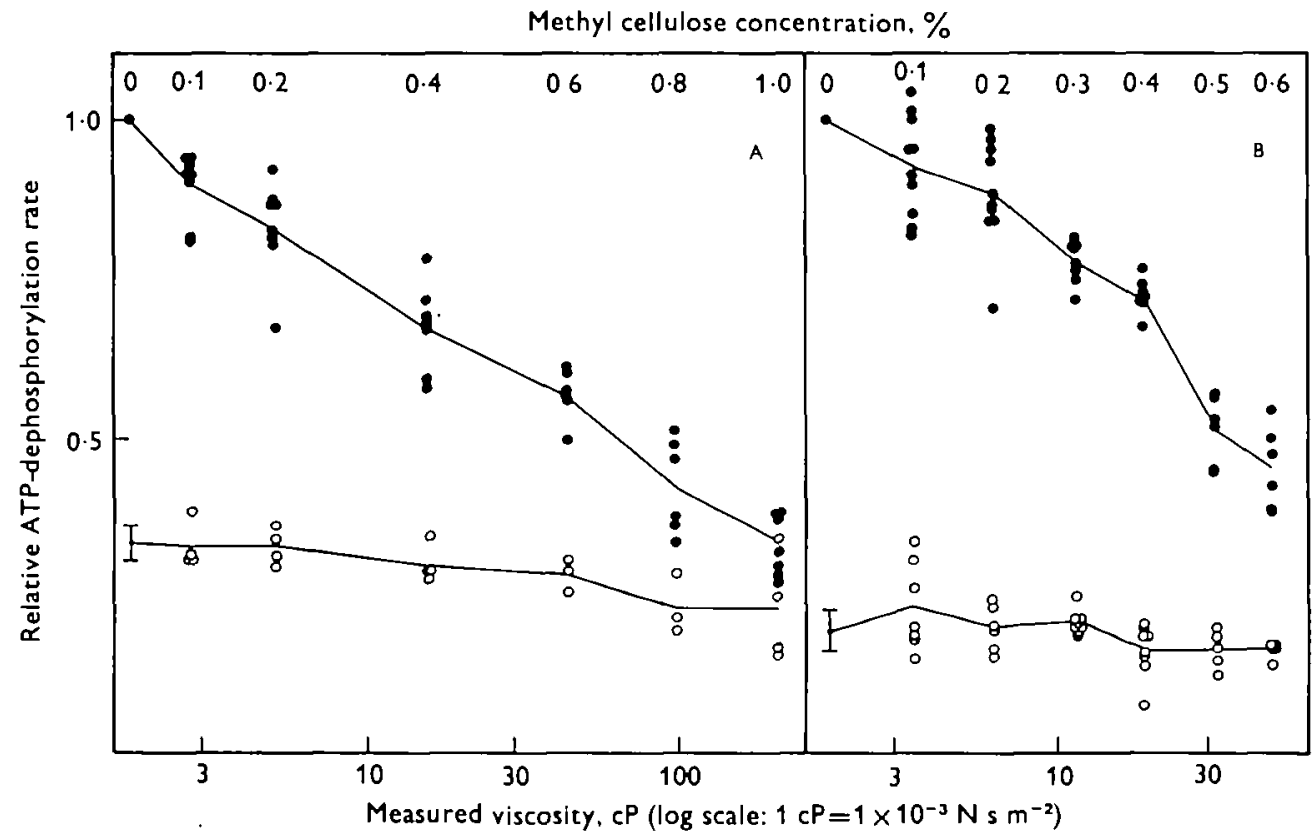

Fig. I. pH-stat measurements of ATP-dephosphorylation by suspensions of Tritondemembranated spermatozoa. For $A$, the reactivation solutions contained $I \cdot 3 \mathrm{~mm}$ $\mathrm{MgSO}_{4}$ and $0.33 \mathrm{~mm} \mathrm{ATP}$; for $\mathrm{B}$, the reactivation solutions contained $3.0 \mathrm{mM}$ $\mathrm{MgSO}_{4}$ and $0.35 \mathrm{~mm}$ ATP. The solid points show measurements from suspensions containing intact spermatozoa. The open points show measurements from suspensions containing broken (A) or trypsin-disintegrated (B) spermatozoa. The lines connect the mean values obtained at each methyl cellulose concentration.

for unbroken sperm preparations represent the rates measured at a particular methyl cellulose concentration, relative to the rate obtained with the same demembranated sperm preparations in the absence of methyl cellulose. For broken sperm preparations, the data points were normalized with respect to the mean of the rates measured with the same sperm sample in the absence of methyl cellulose. The broken sperm preparations routinely showed less than I $\%$ motility, and had a mean relative ATPdephosphorylation rate in the absence of methyl cellulose of $0.33 \pm 0.03$ (standard 
deviation). This rate exceeded 0.40 in the case of results from 4 additional sperm samples, which were discarded and not included in Fig. I.

Visual examination of samples from the experiments using the unbroken sperm preparations in these experiments routinely showed a small fraction of non-motile flagellar fragments and partially motile broken spermatozoa, and few or no intact, non-motile spermatozoa. Our rough estimate of the percentage of normally motile spermatozoa would be about $95 \%$, but we found no way to make an accurate quantitative measurement of this percentage. At methyl cellulose concentrations of $0.6 \%$ or higher, the percentage motility appeared to be less, but it was even more difficult to estimate accurately because the amplitudes of bending were low. With I $\%$ methyl cellulose, and often with $0.8 \%$ methyl cellulose, few of the spermatozoa showed any visible bending unless their heads were attached to the microscope slide, and reliable frequency measurements could not be obtained from the $\mathrm{pH}$-stat samples.

Sperm counts were carried out with 8 sperm samples in order to calculate an absolute ATP dephosphorylation rate. The ATP-dephosphorylation rate for the 22 samples of unbroken spermatozoa in the absence of methyl cellulose had a mean value of $50.3 \mathrm{nM} \mathrm{min}-1$ and a standard deviation of $3.9 \mathrm{nM} \mathrm{min}{ }^{-1}$, for samples corresponding to $2 \cdot 4 \pm 0.2 \times 10^{8}$ spermatozoa. The mean beat frequency of spermatozoa in samples taken from these $\mathrm{pH}$-stat experiments was $20.8 \pm 0.5 \mathrm{~Hz}$. These measurements lead to values of $3.5 \times 10^{-18} \mathrm{~mol}$ of ATP s${ }^{-1}$ per spermatozoon and $\mathrm{r} \cdot 7 \times 10^{-19} \mathrm{~mol}$ of ATP per beat per spermatozoon.

After completing the first series of $\mathrm{pH}$-stat experiments, we were not satisfied that we could distinguish completely between amplitude and frequency effects at intermediate viscosities. Subsequently, we found that the amplitude of the bending waves obtained at intermediate viscosities was sensitive to the $\mathrm{Mg}^{2+}$ ion concentration in the reactivation solutions. A second series of experiments was carried out to examine the effects of $\mathrm{Mg}^{2+}$ ion concentration differences on the rate of ATP-dephosphorylation. These experiments were carried out with broken and unbroken sperm preparations from 8 sperm samples, and all the measurements were made in reactivation solutions containing $0.3 \%$ methyl cellulose. In each experiment, a high-Mg solution containing $3.0 \mathrm{mM} \mathrm{MgSO}_{4}$ and $0.35 \mathrm{~mm}$ ATP was compared with a low-Mg solution. In the high-Mg solution, the reactivated spermatozoa swam regularly with bend angles maintained near $2 \cdot 0$ radians and a mean beat frequency of $15.0 \mathrm{~Hz}$. The $\mathrm{MgSO}_{4}$ and ATP concentrations in the low-Mg solutions varied in different experiments, because we were attempting to match the beat frequencies obtained in the 2 solutions. In the low- $\mathrm{Mg}$ solutions, $\mathrm{MgSO}_{4}$ concentrations ranging from 0.35 to $0.56 \mathrm{~mm}$ and ATP concentrations ranging from 0.35 to $0.45 \mathrm{~mm}$ were used. The mean beat frequency in the low-Mg solutions was $15.4 \mathrm{~Hz}$. In the low-Mg solutions, the spermatozoa showed a wider variation in bend angles, but they were usually in the range of $\mathrm{I} \cdot \mathrm{O}-\mathrm{I} \cdot 5$ radians. The results of these experiments are summarized in Table $\mathrm{I}$.

A third series of $\mathrm{pH}$-stat experiments was carried out with reactivation solutions containing $3.0 \mathrm{mM} \mathrm{MgSO}$ and $0.35 \mathrm{mM} \mathrm{ATP}$, using methyl cellulose concentrations of $0.1,0.2,0.3,0.4,0.5$, and $0.6 \%$. In this series, the ATP-dephosphorylation by non-motile spermatozoa was obtained by disintegrating the spermatozoa with trypsin 
at the end of each pH-stat measurement. The results are shown in Fig. I B in the same style as in Fig. I A. For these experiments, the mean "rate of ATP dephosphorylation for 20 samples in the absence of methyl cellulose was $46.7 \pm 5.0 \mathrm{nM} \mathrm{min}^{-1}$, and the mean beat frequency was $\mathrm{I} 9 . \mathrm{I} \pm 0.8 \mathrm{~Hz}$.

In the absence of methyl cellulose the ATP-dephosphorylation rate measured after the addition of trypsin was $0.19 \pm 0.03$ times the initial rate ( 22 measurements) and the rate measured after the addition of trypsin and trypsin inhibitor was $0.18 \pm$ 0.04 times the initial rate ( 18 measurements). The post-trypsin measurements shown in Fig. I B were all obtained without the addition of trypsin inhibitor.

Table I. Effect of $\mathrm{Mg}^{2+}$ ion concentration on the rate of $A T P$-dephosphorylation by suspensions of demembranated spermatozoa in the presence of $0.3 \%$ methyl cellulose

\begin{tabular}{lcc}
\hline & $\begin{array}{c}\text { ATP-dephosphorylation } \\
\text { rate } \\
\text { nM min }\end{array}$ & $\begin{array}{c}\text { Standard } \\
\text { deviation, } \\
\text { nM min }\end{array}$ \\
\hline Unbroken spermatozoa & & \\
High-Mg & 33.9 & \pm 2.8 \\
Low-Mg & 32.7 & \pm 5.3 \\
Broken spermatozoa & & \\
High-Mg & 13.0 & \pm 2.6 \\
Low-Mg & I4.0 & \pm 3.0 \\
\hline
\end{tabular}

\section{Movement parameters}

The most complete measurements on movement parameters were obtained from experiments carried out independently of the $\mathrm{pH}$-stat experiments, using buffered reactivation solution containing $1 \cdot 3 \mathrm{mM} \mathrm{MgSO}_{4}$ and $0.33 \mathrm{mM}$ ATP. As the viscosity is increased, swimming spermatozoa gradually decrease their beat frequency and wavelength, with little change in bend angle, up to methyl cellulose concentrations of approximately $0.3 \%$. At higher viscosities, there is an increasing percentage of spermatozoa swimming with noticeably reduced bend angles, and at methyl cellulose concentrations above $0.4 \%$, spermatozoa swimming with normal bend angles are only rarely observed. On the other hand, spermatozoa which are beating with the sperm head attached to the microscope slide can maintain normal or greater than normal bend angles at methyl cellulose concentrations up to $\mathrm{I} \cdot 0 \%$.

Measurements of beat frequencies are summarized in Figs. 2 and 3. Data for spermatozoa swimming with near-normal bend angles, at $0-0.4 \%$ methyl cellulose, are shown in Fig. 2; data for spermatozoa swimming with much lower than normal bend angles at $0.3-0.8 \%$ methyl cellulose, and data for attached spermatozoa are shown in Fig. 3. The swimming spermatozoa with low bend angles maintain approximately constant beat frequencies as the viscosity is increased. Their beat frequencies are much more variable, both within a particular sperm preparation and between different sperm samples. Within a particular sperm preparation we sometimes observed that the higher beat frequencies appeared to correspond to the smaller bend angles, but we were unable to obtain quantitative data on these small bend angles to 
confirm this impression. If this correlation is consistently present, it will introduce additional uncertainty into the beat frequency measurements, since the beat frequencies of spermatozoa with larger bend angles were easier to measure, and therefore tended to predominate in the sample we measured.

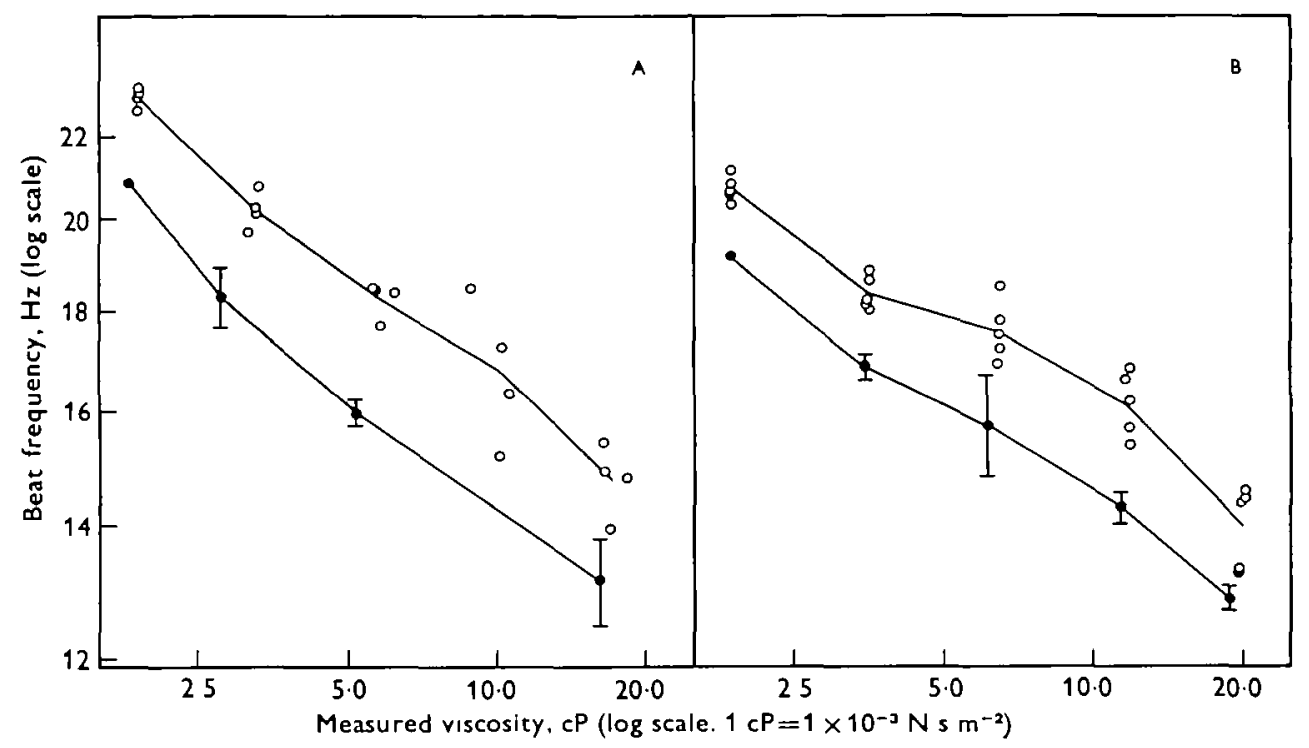

Fig. 2. Beat frequencies of reactivated spermatozoa in solutions containing $0-0.4 \%$ methyl cellulose. For $\mathrm{A}$, the reactivation solutions contained ${ }_{1} \cdot 3 \mathrm{mM} \mathrm{MgSO}_{4}$ and $0.33 \mathrm{~mm}$ ATP; for $\mathrm{B}$, the reactivation solutions contained $3.0 \mathrm{mM} \mathrm{MgSO}$, and $0.35 \mathrm{~mm}$ ATP. The solid points show the mean beat frequency, relative to the beat frequency measured in the absence of methyl cellulose, for spermatozoa in samples from the $\mathrm{pH}$ stat experiments, and standard deviations are shown. The open points show mean beat frequencies for each of several sperm samples studied in the independent experiments. The logarithmic scales used for the ordinate and abscissa in Figs. 2-5 differ by a factor of 4 , so that a slope of $-I \cdot O$ in the figures corresponds to an actual slope of -0.25 for ( $\log$ frequency $/ \log$ viscosity), etc.

Measurements of bend angles and wavelengths from photographs obtained with 2 of these sperm samples are summarized in Figs. 4 and 5. Less complete measurements on a third sample were similar. The swimming spermatozoa photographed for these measurements were all ones which were considered to have near-normal bend angles. We were unable to obtain significant quantitative data from photographs of freely swimming spermatozoa with small bend angles, as these small amplitude bending waves were difficult to resolve and varied greatly between individual spermatozoa, and because these spermatozoa were seldom constrained to swim parallel to a surface without sticking to it. The bend angle is relatively insensitive to small increases in viscosity, but as the viscosity approaches the level where most of the spermatozoa switch to a low-amplitude mode, there is some reduction in the bend angles of the spermatozoa swimming with near-normal bend angles. Although both of these sperm samples responded in a similar way to increased viscosity, they differed in their response to attachment of the sperm head to the microscope slide. One sample showed 


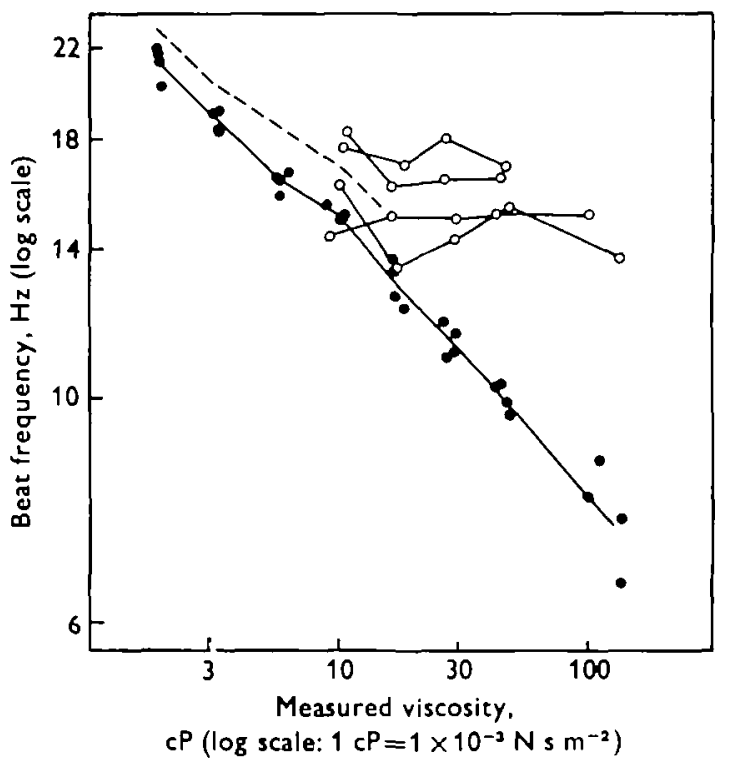

Fig. 3. Beat frequencies of reactivated spermatozoa. The open points show mean beat frequencies for spermatozoa swimming with low bend angles; the points from individual sperm samples are connected by lines. The solid points show mean beat frequencies for spermatozo beating with their heads attached to the microscope slide, accompanied by a line connecting the mean values obtained at each methyl cellulose concentration. The dashed line shows, for comparison, the results obtained with swimming spermatozoa with normal bend angles, from Fig. $2 \mathrm{~A}$.

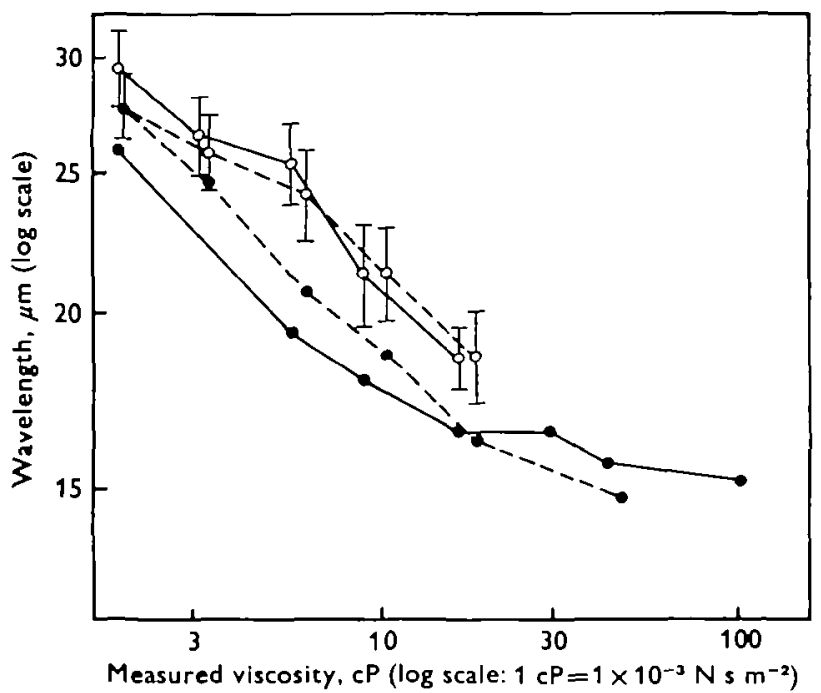

Fig. 4. Wavelength measurements from photographs of 2 different sperm samples, distinguished by solid and dashed lines. The open circles are measurements on swimming spermatozoa with near-normal bend angles; the solid points are measurements on attached spermatozoa. 
little change in mean bend angle or wavelength when attached, but with the other sample, the bend angle of attached spermatozoa was significantly increased while the wavelength of attached spermatozoa was significantly decreased.

Visual observations of spermatozoa swimming with low-amplitude waves at high viscosities indicate that the wavelength continues to decrease uniformly as the viscosity is increased. Bending becomes progressively restricted to the proximal region of the flagellum, so that there is no further increase in the number of visible bends on a flagellum as the viscosity is increased. The bend angles also appear to decrease at higher viscosities, although these are more difficult to estimate visually than the wavelength.

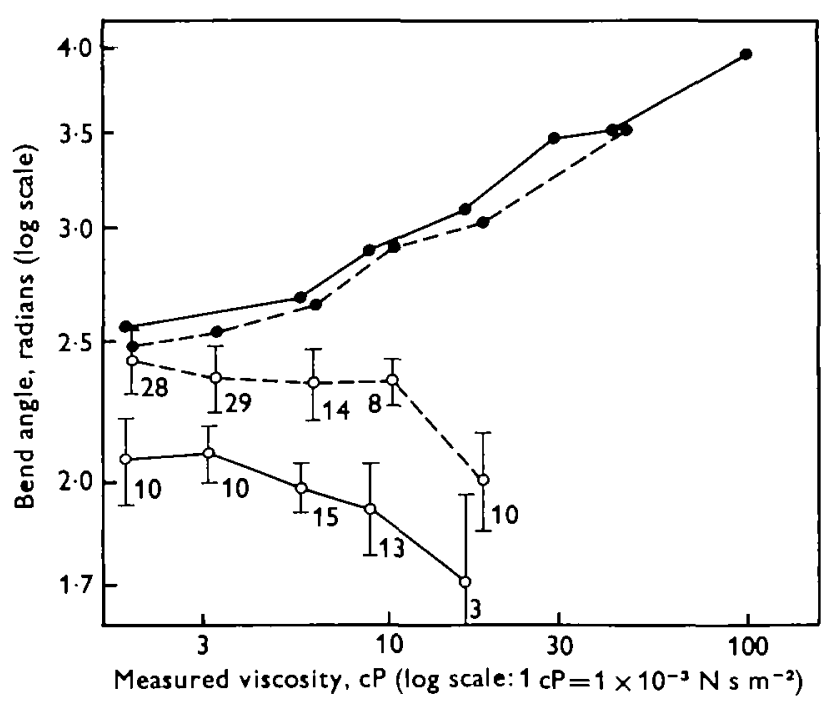

Fig. 5. Bend angle measurements from photographs of 2 sperm samples distinguished by solid and dashed lines as in Fig. 4. The open circles are measurements on swimming spermatozoa, the solid points are measurements on attached spermatozoa. The number of sperm photographs measured is also indicated in this figure, for the swimming spermatozoa. Standard deviations for attached spermatozoa were omitted from these curves to avoid confusion, but they were comparable to those shown for swimming spermatozoa.

At methyl cellulose concentrations near $0.4 \%$, the head of a spermatozoon swimming with low amplitude waves oscillates visibly through an angle of approximately \pm 0.5 radian as the proximal region of the flagellum bends. This contrasts with the spermatozoa swimming with normal bend angles, which appear to maintain a much more constant head orientation. As the viscosity is further increased, the head oscillation appears to be suppressed. These observations imply that although there is a rather marked change in bend angle in the region of $0.4 \%$ methyl cellulose, there may be a much more gradual change in the amplitude of sliding between flagellar microtubules.

Measurements of the total length of the sperm tail in 74 of the photographs used to obtain the data in Figs. 4 and 5 gave a value of $38 \cdot 0 \pm \mathrm{r} \cdot 8 \mu \mathrm{m}$ for the length of the flagellum of $S$. purpuratus spermatozoa. 
Beat frequency measurements for 5 sperm samples at $3.0 \mathrm{mM} \mathrm{MgSO}_{4}$ and $0.35 \mathrm{mM}$ ATP are shown in Fig. $2 \mathrm{~B}$. The beat frequencies obtained in the absence of methyl cellulose are lower than those obtained with $\mathrm{I} \cdot 3 \mathrm{mM} \mathrm{MgSO}_{4}$ (Fig. 2A), but this difference decreases as the methyl cellulose concentration is raised to $0.4 \%$. As a result of this difference in behaviour, the slope of the measurements in Fig. 2 B corresponds more closely to the results obtained in an earlier study with Lytechinus spermatozoa (Brokaw, r975a).

Also shown in Fig. 2 are measurements of beat frequencies of spermatozoa in samples taken from the $\mathrm{pH}$-stat experiments at various methyl cellulose concentrations. These beat frequencies are consistently about 10\% lower than the beat frequencies measured during the independent experiments, but the slopes and shapes of the curves are very similar. The major factor responsible for this difference in beat frequencies appears to be the high sperm concentration used in the pH-stat experiments. At these high sperm concentrations, the beat frequency decreases at a rate of approximately I \% per min, irrespective of the presence of Tris buffer. At the low sperm concentrations used for the independent measurements, the beat frequency remains essentially constant over a period of I $^{-20} \mathrm{~min}$.

Visual observation of bend angles on samples from the $\mathrm{pH}$-stat experiments were in agreement with the independent observations (Fig. 5). In the series of pH-stat experiments using $3.0 \mathrm{mM} \mathrm{MgSO}_{4}$ and $0.35 \mathrm{mM} \mathrm{ATP}$, bend angles remained near 2 radians over the range of $0-0.4 \%$ methyl cellulose, but were much lower at 0.5 and $0.6 \%$ methyl cellulose. Photographs were obtained of spermatozoa in samples taken from two of the $\mathrm{pH}$-stat experiments comparing $\mathrm{o}$ and $0.3 \%$ methyl cellulose solutions, with $3 \mathrm{mM} \mathrm{MgSO}$ and $0.35 \mathrm{~mm}$ ATP. Nine good photographs were obtained from the samples without methyl cellulose, and these gave a mean wavelength of $28.4 \mu \mathrm{m}$ with a standard deviation of $\mathrm{I} \cdot 7 \mu \mathrm{m}$ and a mean bend angle of 2.33 radians with a standard deviation of 0.1 I radians. Eighteen good photographs were obtained from the samples with $0.3 \%$ methyl cellulose, at a measured viscosity of II.7 $\mathrm{cP}$ $\left(\mathrm{I} \cdot \mathrm{I} 7 \times \mathrm{IO}^{-2} \mathrm{~N} \mathrm{~s} \mathrm{~m}^{-2}\right)$. These photographs gave a mean wavelength of $20.3 \mu \mathrm{m}$ with a standard deviation of $2.8 \mu \mathrm{m}$ and a mean bend angle of 2.03 radians with a standard deviation of 0.17 radians. These measurements are consistent with the measurements from the independent experiments shown in Figs. 4 and 5, and confirm that the changes in bend angle in this viscosity range are small compared to the changes in beat frequency and wavelength.

\section{DISCUSSION}

\section{Identification of movenient-coupled ATP-dephosphorylation}

The difference between the ATP-dephosphorylation rates of unbroken and broken sperm samples was identified as a movement-dependent A'TP-dephosphorylation in the original experiments with glycerol-demembranated sea urchin spermatozoa (Brokaw \& Benedict, r968a). In those experiments only a small fraction of the demembranated spermatozoa regained normal motility, and non-motile spermatozoa presumably made a large contribution to the ATP-dephosphorylation rates measured 
with unbroken sperm samples. In our present experiments, using the Tritondemembranation technique developed by Gibbons \& Gibbons (1972), almost all of the spermatozoa can be reactivated, and the contribution of non-motile spermatozoa to the measured ATP-dephosphorylation rates is small. The use of oligomycin to inhibit mitochondrial ATPase activity (Gibbons \& Gibbons, 1972) also decreases the contribution of non-movement-coupled ATP-dephosphorylation to the measured dephosphorylation rates.

Elimination of motility by sperm breakage reduces the ATP-dephosphorylation rate to 0.33 times the rate obtained with motile spermatozoa. In other experiments, we found that inhibition of flagellar bending with $0.1 \mathrm{M} \mathrm{NaHCO}_{3}$ reduced the rate of ATP-dephosphorylation to about $70 \%$ of the value obtained with broken spermatozoa (Brokaw \& Sjmonick, I976). We would then expect that bicarbonate-inhibition of motility would reduce the ATP-dephosphorylation rate to 0.23 times the rate obtained with motile spermatozoa. Elimination of motility by disintegrating the spermatozoa with trypsin reduces the ATP-dephosphorylation rate to $0.18-0.19$ times the rate obtained with motile spermatozoa. Each of these techniques leads to a different estimate for movement-coupled ATP-dephosphorylation, but none of them necessarily distinguish between A'TP-dephosphorylation which is useful for but not tightly coupled to, motility, and unrelated ATPase activity which may be in the spermatozoa.

We have suggested that the difference between the ATP-dephosphorylation rates of broken and bicarbonate-inhibited spermatozoa may represent ATP-dephosphorylation coupled to oscillatory sliding between flagellar tubules, which can occur in the absence of bending only if the flagella are broken (Brokaw \& Simonick, 1976). The difference between the ATP-dephosphorylation rates of bicarbonate-inhibited and trypsin-disintegrated spermatozoa might represent ATP-dephosphorylation coupled to 'isometric' force generation between flagellar tubules, analogous to the ATPase cost of isometric tension generation in muscle. If these speculations are correct, these latter 2 components of ATP-dephosphorylation, present in broken spermatozoa, represent ATP-dephosphorylation which contributes to the generation of movement in unbroken spermatozoa. Trypsin-disintegration might then be a preferable technique for identifying the movement-coupled ATP-dephosphorylation.

Our measurements give an absolute value of $\mathrm{r} \cdot 4 \times \mathrm{IO}^{-19} \mathrm{~mol}$ of ATP per beat per spermatozoon for the movement-coupled ATP-dephosphorylation if trypsin-disintegrated spermatozoa are used, and $\mathbf{I} \cdot \mathrm{I} \times 10^{-19} \mathrm{~mol}$ of ATP per beat per spermatozoon for the movement-coupled ATP-dephosphorylation if broken spermatozoa are used. The latter value is identical to the value calculated in the same manner for Tritonextracted spermatozoa of Colobocentrotus (Gibbons \& Gibbons, 1972). These values also agree well with the value of $1 \cdot 7 \times 10^{-19} \mathrm{~mol}$ of ATP per beat per spermatozoon, obtained by a different method, for the movement-coupled ATP-dephosphorylation by live spermatozoa of Ciona (Brokaw \& Benedict, I968b), especially since Ciona spermatozoa are about $20 \%$ longer than Strongylocentrotus purpuratus spermatozoa. 


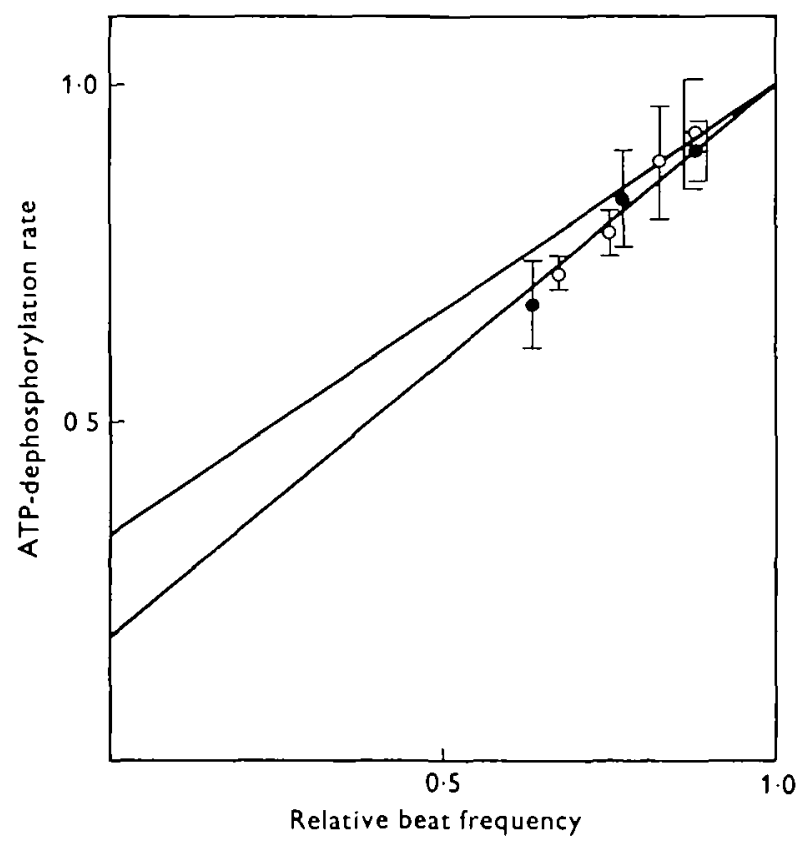

Fig. 6. Comparison of relative ATP-dephosphorylation rate and relative beat frequency for spermatozoa swimming with near-normal bend angles. The solid points are from the experiments with reactivation solutions containing $\mathrm{I} \cdot 3 \mathrm{~mm} \mathrm{MgSO}_{4}$ and $0.33 \mathrm{mM}$ ATP; the open points are from the experiments with reactivation solutions containing $3.0 \mathrm{~mm} \mathrm{MgSO}$ and $0.35 \mathrm{~mm}$ ATP. Standard deviations for the ATPdephosphorylation rate measurements are indicated. The lines indicate the results expected if movement-coupled ATP-dephosphorylation is proportional to beat frequency, using 2 different estimates of the movement-coupled component of ATPdephosphorylation.

\section{Effects of increased viscosity}

Measurements of ATP-dephosphorylation and beat frequency for reactivated spermatozoa swimming in solutions containing $0-0.4 \%$ methyl cellulose are compared in Fig. 6. The results are in agreement with the initial observations shown in fig. 4 of Brokaw \& Benedict (1968a) indicating that there is a linear relationship between ATP-dephosphorylation and beat frequency when the beat frequency is altered by changes in viscosity. The present results are more precise, and we can also now state with confidence that the decreased ATP-dephosphorylation is not significantly influenced by changes in bend angle or the percentage of motile spermatozoa. Two reference lines are shown in Fig. 6, to indicate the results expected if the movement-coupled ATP-dephosphorylation is proportional to beat frequency. The upper line, which intersects the ordinate at a relative ATP-dephosphorylation rate of 0.33 , corresponds to the result expected if the difference between the ATP-dephosphorylation rates of unbroken and broken sperm samples is taken as a measure of movement-coupled ATP-dephosphorylation. The lower line, which intersects the ordinate at a relative ATP-dephosphorylation rate of 0.185 , corresponds to the result expected if the difference between the ATP-dephosphorylation rates of intact and trypsin- 
disintegrated sperm samples is taken as a measure of movement-coupled ATPdephosphorylation. The results correspond somewhat more closely to the lower line. It might be argued that the deviation of the results from the upper line is caused by an effect of increased viscosity on other variables such as bend angle which might influence the rate of ATP-dephosphorylation. We were unable to find any evidence that such small changes in bend angle could influence the ATP-dephosphorylation rate, but the variability of our results is too great to exclude this possibility completely.

The further decrease in the rate of ATP-dephosphorylation which occurs with methyl cellulose concentrations of $0.5-1.0 \%$ cannot be ascribed solely to a reduction in beat frequency, as we were unable to measure a consistent decrease in beat frequency for swimming spermatozoa at these higher methyl cellulose concentrations. The decreased rate of ATP-dephosphorylation observed at high methyl cellulose concentrations may be related to the severe reduction in bend angle and the restriction of bending to the basal portion of the flagellum which is observed at high methyl cellulose concentrations.

Increased viscosities also cause a decrease in the wavelength of the flagellar bending waves. Comparison of the data in Figs. I and 4 suggests, in fact, that the decreases in movement-coupled ATP-dephosphorylation follow the decrease in wavelength more closely than any other single parameter of movement. Nevertheless, we do not believe that the movement-coupled ATP-dephosphorylation is determined by the wavelength. In other work, the wavelength has been shown to decrease gradually with increasing ATP concentration (Brokaw, 1975a) while the movement-coupled ATPdephosphorylation increases with ATP concentration (Brokaw \& Benedict, i968a; Gibbons \& Gibbons, 1972). Active sliding between flagellar tubules has been clearly implicated as the source of active flagellar bending (Summers \& Gibbons, 1971). The rate of active sliding at a particular point on a flagellum is related to both the beat frequency and the bend angle, but is not related to the wavelength, which merely determines the phase relationships of oscillatory sliding at different points along the length of a flagellum.

In the context of models for cross-bridge driven active sliding between flagellar tubules (Brokaw, $1975 b, 1976$ ), ATP turnover should be determined most directly by the rate of sliding, and therefore be proportional to the product of beat frequency and bend angle. Our results are more or less consistent with this model, but the evidence for a relationship between bend angle and ATP-dephosphorylation is still unsatisfactory. In particular, we were unable to obtain such evidence from our experiments comparing ATP-dephosphorylation rates at high and low $\mathrm{MgSO}_{4}$ concentrations (Table $\mathrm{I}$ ), even though a substantial difference in bend angle was observed. However, there was also an observable difference in the amount of angular oscillation of the sperm head, so that the bend angle is not a sufficient indicator of the amplitude of active sliding. Further studies of this problem are required.

\section{Energy limitations}

The data on movement parameters for spermatozoa reactivated in solutions containing $1.3 \mathrm{mM} \mathrm{MgSO}_{4}$ and $0.33 \mathrm{mM}$ ATP have been used to calculate the rate of 
energy expenditure against the viscous resistance of the surrounding fluid, using equation (6) of Brokaw ( $1975 c$ ). In the absence of methyl cellulose, at a beat frequency of $20.8 \mathrm{~Hz}$, the energy expenditure of a swimming spermatozoon is $0.019-$ $0.028 \mathrm{pJ} \mathrm{s}^{-1}$. The range of values represents uncertainty about the correct value which should be used for the viscous drag coefficient, $C_{L}$. Recent hydrodynamic analysis suggests that the values used previously (Brokaw, I965, etc.) are too low and should be increased by about 50\% (Katz \& Blake, 1975; Brokaw \& Gibbons, 1975). The calculated rates of energy expenditure are also approximate, because they are based on formulae for bending waves propagated along long filaments and do not take into account end effects and the energy required to move the sperm head. However, these approximations appear to be relatively less serious. Using the value of $1 \cdot 4 \times 10^{-19} \mathrm{~mol}$ of ATP per beat then indicates that the energy conversion from ATP under these conditions corresponds to $6 \cdot 4-9 \cdot 6 \mathrm{~kJ} \mathrm{~mol}^{-1}$. The free energy change for ATPdephosphorylation under the conditions of these experiments is not accurately known, but is presumably in the range of $30-50 \mathrm{~kJ} \mathrm{~mol}^{-1}$.

When the viscosity is increased, a constant rate of energy expenditure would be maintained if the bend angle remains constant and if both beat frequency and wavelength decrease in inverse proportion to the $4^{\text {th }}$ root of the viscosity (cf. equation (6) of Brokaw, 1975c). This decrease would correspond to slopes of $-\mathrm{I} \cdot 0$ in Figs. 2 and 4 ; the data in these figures show smaller decreases. Consequently, the energy expenditure in $0.4 \%$ methyl cellulose increases to $0.024-0.036 \mathrm{pJ} \mathrm{s}^{-1}$, while the movement-coupled ATP-dephosphorylation decreases. The energy conversion under these conditions must therefore increase to $13 \cdot 7-20 \cdot 6 \mathrm{~kJ} \mathrm{~mol}^{-1}$. If these changes continue in the same way when the viscosity is increased further, at some point the energy conversion required to overcome viscous resistance will reach a limit, and some other change in behaviour must occur. This energy limitation may therefore be responsible for the decrease in bend angle which occurs at higher methyl cellulose concentrations. If the ATP-dephosphorylation rate is also proportional to the amplitude of sliding, a small decrease in bend angle will not stabilize the energy balance. The drastic change in bend angle under these conditions may therefore provide some support for the idea that the rate of ATP-dephosphorylation is also related to the amplitude of sliding.

The beat frequency and wavelengths of attached spermatozoa are lower than for swimming spermatozoa. Although the calculation of energy expenditure for attached spermatozoa and at high methyl cellulose concentration is subject to additional uncertainties (cf. Brokaw, I 966), the calculated energy expenditure for attached spermatozoa appears to remain in the range of $0.013-0.03 \mathrm{pJ} \mathrm{s}^{-1}$, even at high viscosities. If, in addition, the rate of ATP-dephosphorylation is proportional to the product of beat frequency and bend angle, there will be relatively little decrease in the rate of ATP-dephosphorylation by attached spermatozoa at high viscosities. As a result of both of these effects, the energy conversion limitation which appears to limit the movement of swimming spermatozoa would not be a significant limitation on the movement of attached spermatozoa. The ability of attached spermatozoa to maintain normal or larger than normal bend angles at high viscosities may therefore be an 
additional argument for a dependence of ATP-dephosphorylation on shear amplitude as well as beat frequency.

This work was supported by USPHS grant GM-I87Ir.

\section{REFERENCES}

BRokAw, C. J. (1965). Non-sinusoidal bending waves of sperm flagella. F. exp. Biol. 43, 155-169. Brokal, C. J. (I966). Effects of increased viscosity on the movements of some invertebrate spermatozoa. F. exp. Biol. 45, I I 3-1 39 .

BrokAlv, C. J. (I975a). Effects of viscosity and ATP concentration on the movement of reactivated sea urchin sperm flagella. 7. exp. Biol. 62, 701 719 .

Brokaw, C. J. (1975b). Cross-bridge behaviour in a sliding filament model for flagella. In Molecules and Cell Movement (ed. S. Inoue \& R. E. Stephens), pp. 165-179. New York: Raven Press.

Brokaw, C. J. (1975c). Spermatozoan motility: A biophysical survey. In The Biology of the Male Gamete (ed. J. G. Duckett \& P. A. Racey), pp. 423-439. Biol. J. Linn. Soc. 7, Suppl. I. London: Academic Press.

Brokaw, C. J. (1976). Computer simulation of flagellar movement. IV. Properties of an oscillatory two-state cross-bridge model. Biophys. 7. 16, 1029-1041.

Brokaw, C. J. \& Benedict, B. (ig68a). Mechanochemical coupling in flagella. I. Movementdependent dephosphorylation of ATP by glyce1 inated spermatozoa. Archs Biochem. Biophys. 125, 770-778,

Brokaw, C. J. \& Benedict, B. (1968b). Mechanochemical coupling in flagella. II. Effects of viscosity and thiourea on metabolism and motility of Ciona spermatozoa. F. gen. Physiol. 52, 283-299.

Brokaw, C. J. \& Gibrons, I. R. (1975). Mechanisms of movement of flagella and cilia. In Swimming and Flying in Nature, vol. I (ed. T. Y. Wu, C. J. Brokaw \& C. Brennan), pp. 89-126. New York: Plenum Publishing.

Brokaw, C. J., Josslin, R. \& Bobrow, L. (1974). Calcium ion regulation of flagellar beat symmetry in reactivated sea urchin spermatozoa. Biochem. biophys. Res. Commun. 58, 795800 .

Brokaw, C. J. \& Simonick, T. F. (I976). $\mathrm{CO}_{2}$ regulation of the amplitude of flagellar bending. In Cell Motility (ed. R. Goldman, R. Pollard \& J. Rosenbaum). Cold Spring Harbor Laboratory. (In Press.)

Gibbons, B. H. \& Gibbons, I. R. (I972). Flagellar movement and adenosine triphosphatase activity in sea urchin sperm extracted with Triton X-100. F. Cell Biol. 54, 75-97.

Gibbons, I. R. \& Fronk, E. (1972). Some properties of bound and soluble dynein from sea urchin sperm flagella. $\mathcal{F}$. Cell Biol. 54, 365-38 $\mathrm{I}$.

Katz, D. F. \& Blake, J. (1975). Flagellar motions near walls. In Swimming and Flying in Nature, vol. I (ed. T. Y. Wu, C. J. Brokaw \& C. Brennan), pp. 173-184. New York: Plenum Publishing.

LiNCK, R. W. (I973). Chemical and structural differences between cilia and flagella from the lamellibranch mollusc, Aequipecten irradians. $\mathcal{F}$. Cell Sci. 12, 95 I-98 I.

OGawa, K. (1973). Studies on flagellar ATPase from sea urchin spermatozoa. II. Effect of trypsin digestion on the enzyme. Biochim. biophys. Acta 292, 514-525.

Summers, K. E. \& Gibrons, I. R. (1971). Adenosine-triphosphate-induced sliding of tubules in trypsin-treated flagella of sea urchin sperm. Proc. natn. Acad. Sci. U.S.A. 68, 3092-3096.

(Received 26 fuly 1976) 